\title{
Design of Optimal Output Regulators for Dual-Rate Linear Discrete-Time Systems Based on the Lifting Technique
}

\author{
Yujian Guo and Fucheng Liao \\ School of Mathematics and Physics, University of Science and Technology Beijing, Beijing 100083, China \\ Correspondence should be addressed to Fucheng Liao; fcliao@ustb.edu.cn
}

Received 22 January 2016; Accepted 2 June 2016

Academic Editor: Qingling Zhang

Copyright ( 2016 Y. Guo and F. Liao. This is an open access article distributed under the Creative Commons Attribution License, which permits unrestricted use, distribution, and reproduction in any medium, provided the original work is properly cited.

\begin{abstract}
A design strategy of optimal output regulators for dual-rate discrete-time systems, whose output sampling period is an integer multiple of the input updating period, is proposed. At first, by using the discrete lifting technique, the dual-rate discrete-time system is converted to a single-rate augmented system in form and the lifted state-space model is constructed. Correspondingly, the performance index of the original system is modified to the performance index of the single-rate augmented system. And the original problem is transformed into an output regulation problem for the augmented system. Then, according to the optimal regulator theory, an optimal output regulator for the dual-rate discrete-time system is derived. In the meantime, the existence conditions of the optimal output regulator are discussed. Finally, a numerical example is included to illustrate the effectiveness of the proposed method.
\end{abstract}

\section{Introduction}

Sampling systems are obtained by discretization of a continuous signal for an actual system. For sampling systems, multirate systems arise when the components of the same system have several different sampling rates [1]. In many complex systems, it is unrealistic or sometimes impossible to sample all the physical signals uniformly at one single rate. For example, in chemical industrial process control, the output sampling rate is much slower than the input updating rate because the control output (such as measuring gas molecular weight, etc.) is obtained from laboratory analysis. As the input and the output are sampled in two different sampling periods, the system is often described as a dual-rate system [2, 3]. The dual-rate system is a special and simple case of multirate systems. In recent years, multirate systems in petrochemical processes [3], hard disk drives [4], and optimal filtering [5] have obtained full application. At the same time, multirate systems have also gained numerous theoretical developments in predictive control [6], stabilization [7], repetitive control [8], robust control [9], and so on.

An optimal output regulator is designed by using linear quadratic regulator theory. The output regulation problem aims to find the optimal control law that can minimize the sum of the dynamic deviation of output and the dissipated energy of the control variables with the given weight [10]. Research based on linear quadratic regulator theory for multirate system has always been the focus. By solving the linear quadratic regulation problem, a new adaptive technique is proposed for the control of the temperature in a greenhouse in [11]. A time-invariant, single-rate design approach for a multirate optimal regulator is presented, and, as an example, a typical problem in the research of control, the control of an inverted pendulum on a cart, is studied in [12]. In addition, the lifting technique is a standard tool to handle multirate systems in [2]. By using the lifting technique, the multirate system can be converted into a single-rate system. Thus, the control problem for multirate systems can be solved by applying the methods of single-rate systems.

In this regard, preview control based on the optimal regulation theory for discrete-time multirate systems has produced very good research results recently [13-15]. An optimal preview controller design method for discrete-time multirate input systems was proposed in [13]. References $[14,15]$ applied this method to descriptor systems; the optimal preview control problem for discrete-time descriptor causal systems in a multirate setting and discrete-time descriptor noncausal multirate systems was studied, separately. But 
the studies on designing the optimal output regulator for multirate systems were relatively few. Reference [16] studied the robustness of optimal output regulators based on multirate sampling of plant output. Reference [17] introduced the design problem of the optimal output regulator for discretetime descriptor causal multirate input systems.

On the basis of $[16,17]$ and by using the method of [13-15], this paper studies the design problem of optimal output regulators for dual-rate discrete-time systems whose output sampling period is an integer multiple of the input updating period. The basic method is as follows. First, by using the lifting technique, the normal dual-rate system is converted to a single-rate augmented system. Second, by transformation, this problem becomes an optimal control problem of the augmented system. Finally, returning to the original system, an optimal output regulator for the dual-rate discrete-time system is obtained. Furthermore, the stabilizability and detectability of the single-rate augmented system are discussed, and their rigorous mathematical proofs are given. The following lemmas will be used in this paper (see $[18,19])$.

Lemma 1 (PBH test). ( $A, B)$ is stabilizable if and only if the matrix $[\lambda I-A B]$ has full row rank, where $\lambda$ is a complex number satisfying $|\lambda| \geq 1 ;(C, A)$ is detectable if and only if the matrix $\left[\begin{array}{c}\lambda I-A \\ C\end{array}\right]$ has full column rank, where $\lambda$ is a complex number satisfying $|\lambda| \geq 1$.

Lemma 2. $(A, B)$ is stabilizable if and only if $w^{T} A=\lambda w^{T}$; then $w^{T} B \neq 0$, where $\lambda$ is any eigenvalue of $A,|\lambda| \geq 1$, and $w$ is the corresponding left eigenvector.

\section{Description and Related Assumptions}

Consider the following linear discrete-time system:

$$
\begin{aligned}
x(k+1) & =A x(k)+B u(k), \\
y(k) & =C x(k),
\end{aligned}
$$

where $x(k) \in R^{n}, u(k) \in R^{r}$, and $y(k) \in R^{m}$ represent the state vector, the control input vector, and the output vector, respectively. $A, B, C$ are known constant matrices with appropriate dimensions.

For system (1), we introduce the following assumptions:

(A1) The state vector $x(k)$ and output vector $y(k)$ can only be measured at $k=i N(i=0,1,2, \ldots)$, where $N$ is a positive integer.

(A2) The input vector $u(k)$ can only be refreshed at $k=$ $i N_{1}(i=0,1,2, \ldots)$, where $N_{1}$ is a positive integer.

Remark 3. If (A1) and (A2) hold, the system is dual-rate sampled. That is, the state vector $x(k)$ and output vector $y(k)$ can be measured once during every $N$ sampling interval. The input vector $u(k)$ can only be refreshed once during every $N_{1}$ sampling interval.

(A3) Assume $N / N_{1}=p$ in this paper, where $p$ is a positive integer and $p>1$.
Suppose the system has zero-order-hold, that is

(A4) $u\left(i N_{1}+q\right)=u\left(i N_{1}\right), q=0,1,2, \ldots, N_{1}-1$.

(A5) $(A, B)$ is stabilizable and $1+\lambda+\cdots+\lambda^{N_{1}-1} \neq 0$, where $\lambda$ is any eigenvalue of $A$ and $|\lambda| \geq 1$.

Remark 4. (A5) guarantees the existence of the state feedback during the design of the optimal output regulator.

(A6) $(C, A)$ is detectable.

We introduce the quadratic performance index function for system (1):

$$
J=\sum_{k=0}^{\infty}\left[y^{T}(k) F y(k)+u^{T}(k) H u(k)\right],
$$

where the weight matrices satisfy $F>0, H>0$.

We would like to design an optimal output regulator for system (1) with dual-rate setting under the performance index (2).

\section{Design of the Optimal Output Regulator}

In this section, the optimal output regulator for system (1) with a dual-rate setting is obtained.

3.1. Derivation of the Lifting System. Based on the multirate study methods, the dual-rate discrete-time system is converted into a single-rate augmented system in form by using the lifting technique. Lifting technique is a typical approach to multirate control. By using this technique, a fast-rate signal can be mapped to a slow-rate signal with increased dimensionality. While this operation maps a fast-rate signal to a slow-rate signal, the inverse operation maps a lifted signal to a fast-rate signal in $[20,21]$.

Next, a lifted state-space model is constructed.

First, the lifting technique is applied to the input vectors. According to (A2), (A3), and (A4), the input vector $u(k)$ of system (1) can be input at $k=i N_{1}(i=0,1,2, \ldots)$ and $u\left(i N_{1}+\right.$ $q)=u\left(i N_{1}\right)\left(q=0,1,2, \ldots, N_{1}-1\right)$. Noticing $N=p N_{1}$, we have

$$
\begin{gathered}
x(i N+1)=A x(i N)+B u(i N), \\
x(i N+2)=A x(i N+1)+B u(i N), \\
\vdots \\
x\left(i N+N_{1}\right)=A x\left(i N+N_{1}-1\right)+B u(i N), \\
x\left(i N+N_{1}+1\right)=A x\left(i N+N_{1}\right)+B u\left(i N+N_{1}\right), \\
x\left(i N+N_{1}+2\right)=A x\left(i N+N_{1}+1\right)+B u\left(i N+N_{1}\right),
\end{gathered}
$$




$$
\begin{aligned}
& x\left(i N+2 N_{1}\right)=A x\left(i N+2 N_{1}-1\right)+B u\left(i N+N_{1}\right), \\
& \vdots \\
& x\left(i N+(p-1) N_{1}+1\right)=A x\left(i N+(p-1) N_{1}\right) \\
& +B u\left(i N+(p-1) N_{1}\right), \\
& x\left(i N+(p-1) N_{1}+2\right)=A x\left(i N+(p-1) N_{1}+1\right) \\
& +B u\left(i N+(p-1) N_{1}\right), \\
& \quad \vdots \\
& x((i+1) N)=A x(i N+N-1) \\
& +B u\left(i N+(p-1) N_{1}\right),
\end{aligned}
$$$$
(i=0,1,2, \ldots) .
$$

Second, we apply the lifting technique to the state vectors and output vectors. According to (A1), the state vectors can only be measured at $k=i N(i=0,1,2, \ldots)$, where $N$ is a positive integer. That is, the state vector $x(k)$ cannot be used in the state feedback if $k \neq i N$.

Using the first group of equations of (3), we get

$$
\begin{aligned}
x(i N+1) & =A x(i N)+B u(i N), \\
x(i N+2) & =A x(i N+1)+B u(i N) \\
& =A^{2} x(i N)+(A B+B) u(i N) .
\end{aligned}
$$

By that analogy, we have

$$
\begin{aligned}
x\left(i N+N_{1}\right)= & A x\left(i N+N_{1}-1\right)+B u(i N) \\
= & A^{N_{1}} x(i N) \\
& +\left(A^{N_{1}-1} B+\cdots+B\right) u(i N) .
\end{aligned}
$$

Continuing the process of lifting by using the second group of equations of (3) and (5), we get

$$
\begin{aligned}
x\left(i N+N_{1}+1\right)= & A x\left(i N+N_{1}\right)+B u\left(i N+N_{1}\right) \\
= & A^{N_{1}+1} x(i N) \\
& +\left(A^{N_{1}} B+\cdots+A B\right) u(i N) \\
& +B u\left(i N+N_{1}\right), \\
x\left(i N+N_{1}+2\right)= & A x\left(i N+N_{1}+1\right)+B u\left(i N+N_{1}\right) \\
= & A^{N_{1}+2} x(i N) \\
& +\left(A^{N_{1}+1} B+\cdots+A^{2} B\right) u(i N) \\
& +(A B+B) u\left(i N+N_{1}\right) ;
\end{aligned}
$$

then we have

$$
\begin{aligned}
x(i N & \left.+2 N_{1}\right) \\
= & A^{2 N_{1}} x(i N) \\
& +\left(A^{2 N_{1}-1} B+A^{2 N_{1}-2} B+\cdots+A^{N_{1}} B\right) u(i N) \\
& +\left(A^{N_{1}-1} B+A^{N_{1}-2} B+\cdots+B\right) u\left(i N+N_{1}\right) .
\end{aligned}
$$

By using the other equations of (3), we continue lifting and obtain

$$
\begin{aligned}
x(i N & +N) \\
= & A^{N} x(i N)+\left(A^{N-1} B+\cdots+A^{N-N_{1}} B\right) u(i N) \\
& +\left(A^{N-N_{1}-1} B+\cdots+A^{N-2 N_{1}} B\right) u\left(i N+N_{1}\right) \\
& +\left(A^{N-2 N_{1}-1} B+\cdots+A^{N-3 N_{1}} B\right) u\left(i N+2 N_{1}\right) \\
& +\cdots+\left(A^{N_{1}-1} B+\cdots+B\right) u\left(i N+(p-1) N_{1}\right) .
\end{aligned}
$$

That is,

$$
x((i+1) N)=A^{N} x(i N)+\left[\sum_{j=1}^{N_{1}} A^{N-j} B \sum_{j=1}^{N_{1}} A^{N-N_{1}-j} B \cdots \sum_{j=1}^{N_{1}} A^{N-(p-1) N_{1}-j} B\right]\left[\begin{array}{c}
u(i N) \\
u\left(i N+N_{1}\right) \\
\vdots \\
u\left(i N+(p-1) N_{1}\right)
\end{array}\right] .
$$

We introduce the vectors as follows:

$$
\begin{aligned}
& \tilde{x}(i)=x(i N), \\
& \tilde{u}(i)=\left[\begin{array}{c}
u(i N) \\
u\left(i N+N_{1}\right) \\
\vdots \\
u\left(i N+(p-1) N_{1}\right)
\end{array}\right] .
\end{aligned}
$$

Then (9) can be written as

$$
\widetilde{x}(i+1)=\widehat{A} \widetilde{x}(i)+\widehat{B} \widetilde{u}(i),
$$

where

$$
\begin{gathered}
\widehat{A}=A^{N}, \\
\widehat{B}=\left[\begin{array}{llll}
B_{1} & B_{2} & \cdots & B_{p}
\end{array}\right],
\end{gathered}
$$




$$
\begin{gathered}
B_{1}=\sum_{j=1}^{N_{1}} A^{N-j} B=A^{N-N_{1}} B_{p}, \\
B_{2}=\sum_{j=1}^{N_{1}} A^{N-N_{1}-j} B=A^{N-2 N_{1}} B_{p}, \\
\vdots \\
B_{p}=\sum_{j=1}^{N_{1}} A^{N-(p-1) N_{1}-j} B=A^{N_{1}-1} B+A^{N_{1}-2} B+\cdots+B .
\end{gathered}
$$

Correspondingly, the output equation is

$$
\begin{gathered}
y(i N)=C x(i N), \\
y(i N+1)=C x(i N+1), \\
\vdots \\
y\left(i N+N_{1}-1\right)=C x\left(i N+N_{1}-1\right),
\end{gathered}
$$$$
\left[\begin{array}{c}
y(i N) \\
y(i N+1) \\
\vdots \\
y\left(i N+N_{1}-1\right) \\
\hline y\left(i N+N_{1}\right) \\
y\left(i N+N_{1}+1\right) \\
\vdots \\
y\left(i N+2 N_{1}-1\right) \\
\vdots \\
y\left(i N+(p-1) N_{1}+1\right) \\
\vdots \\
y(i N+N-1)
\end{array}\right]=\left[\begin{array}{c}
C \\
C A \\
\vdots \\
C A^{N_{1}-1} \\
C A^{N_{1}} \\
C A^{N_{1}+1} \\
\vdots \\
C A^{2 N_{1}-1} \\
\vdots \\
C A^{(p-1) N_{1}} \\
C A^{(p-1) N_{1}+1} \\
\vdots \\
C A^{N-1}
\end{array}\right] x(i N)
$$

$$
\begin{gathered}
y\left(i N+N_{1}\right)=C x\left(i N+N_{1}\right), \\
y\left(i N+N_{1}+1\right)=C x\left(i N+N_{1}+1\right), \\
\vdots \\
y\left(i N+2 N_{1}-1\right)=C x\left(i N+2 N_{1}-1\right), \\
\vdots \\
y\left(i N+(p-1) N_{1}\right)=C x\left(i N+(p-1) N_{1}\right), \\
x\left(i N+(p-1) N_{1}+1\right)=C x\left(i N+(p-1) N_{1}+1\right),
\end{gathered}
$$

$$
\begin{aligned}
y(i N+N-1)=C x(i N+N-1), & \\
& (i=0,1,2, \ldots) .
\end{aligned}
$$

Similarly, by using the lifting technique, the output equation can be written as

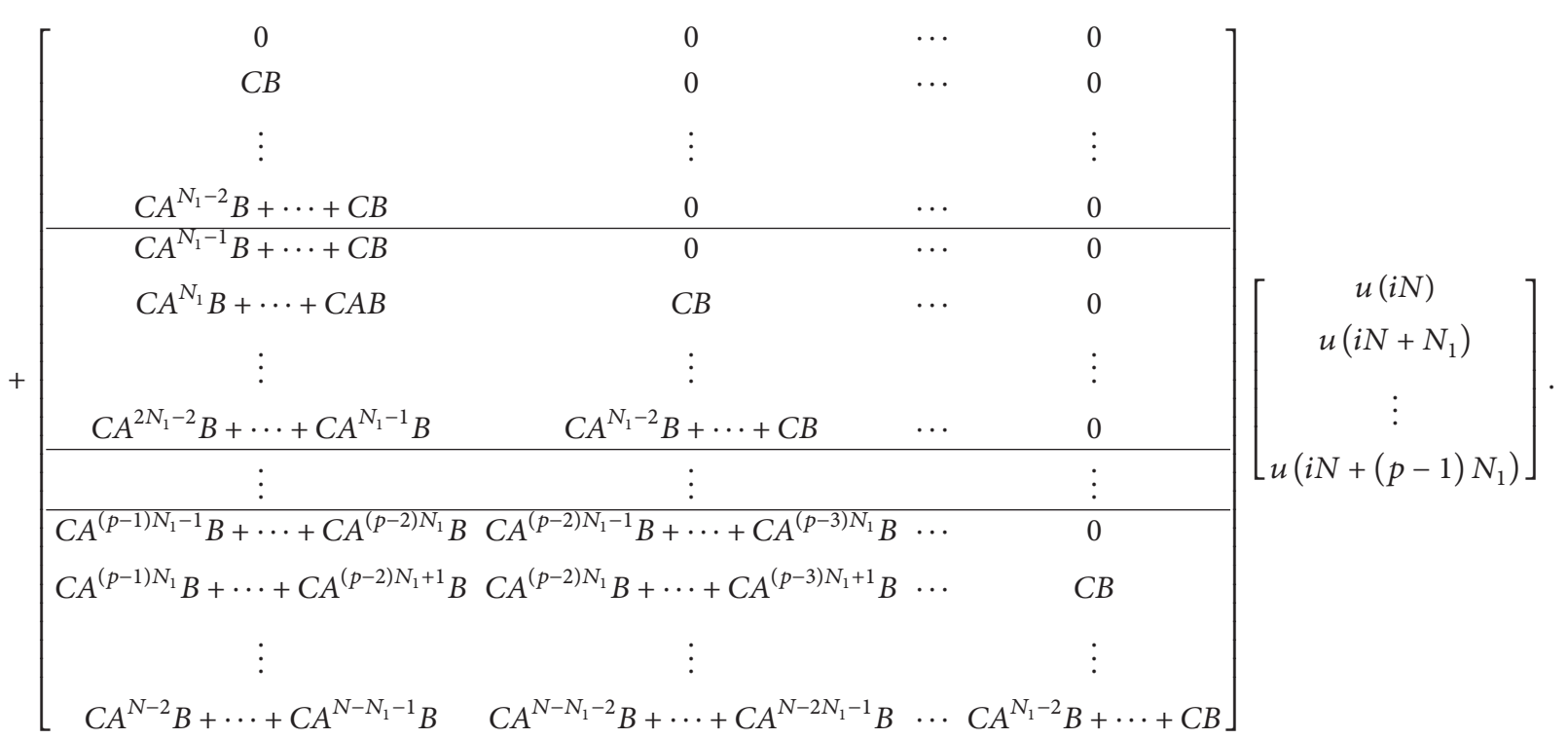


Introduce the vectors as follows:

$$
\tilde{y}(i)=\left[\begin{array}{c}
y(i N) \\
y(i N+1) \\
\vdots \\
y\left(i N+N_{1}-1\right) \\
y\left(i N+N_{1}\right) \\
y\left(i N+N_{1}+1\right) \\
\vdots \\
y\left(i N+2 N_{1}-1\right) \\
\vdots \\
\hline y\left(i N+(p-1) N_{1}\right) \\
y\left(i N+(p-1) N_{1}+1\right) \\
\vdots \\
y(i N+N-1)
\end{array}\right] \in R^{N m} .
$$

Letting

$$
\begin{aligned}
& C_{11}=\left[\begin{array}{c}
C \\
C A \\
\vdots \\
C A^{N_{1}-1}
\end{array}\right] \\
& D_{11}=\left[\begin{array}{c}
0 \\
C B \\
\vdots \\
C A^{N_{1}-2} B+\cdots+C B
\end{array}\right] \text {, } \\
& \widehat{C}=\left[\begin{array}{c}
C_{11} \\
C_{11} A^{N_{1}} \\
\vdots \\
C_{11} A^{(p-1) N_{1}}
\end{array}\right], \\
& \widehat{D}=\left[\begin{array}{cccc}
D_{11} & 0 & \cdots & 0 \\
C_{11} B_{p} & D_{11} & \cdots & 0 \\
\vdots & \vdots & \ddots & \vdots \\
C_{11} A^{(p-2) N_{1}} B_{p} & C_{11} A^{(p-3) N_{1}} B_{p} & \cdots & D_{11}
\end{array}\right] \text {, }
\end{aligned}
$$

(14) can be written as

$$
\tilde{y}(i)=\widehat{C} \tilde{x}(i)+\widehat{D} \tilde{u}(i) .
$$

Combine (11) and (17) to produce

$$
\begin{aligned}
\widetilde{x}(i+1) & =\widehat{A} \widetilde{x}(i)+\widehat{B} \widetilde{u}(i), \\
\tilde{y}(i) & =\widehat{C} \widetilde{x}(i)+\widehat{D} \widetilde{u}(i),
\end{aligned}
$$

where $\widehat{A}, \widehat{B}, \widehat{C}$, and $\widehat{D}$ are as mentioned above.
Now we have successfully transformed the discrete-time system (1) with a dual-rate setting into the single-rate system (18).

3.2. Design Method of the Optimal Output Regulator. Notice that the performance index (2) for system (1) can be represented by relevant vectors in system (18) as follows:

$$
\begin{aligned}
J & =\sum_{k=1}^{\infty}\left[y^{T}(k) F y(k)+u^{T}(k) H u(k)\right] \\
& =\sum_{i=0}^{\infty} \sum_{j=0}^{N-1}\left[y^{T}(i N+j) F y(i N+j)\right. \\
& \left.+u^{T}(i N+j) H u(i N+j)\right]=\sum_{i=0}^{\infty}\left[\widetilde{y}^{T}(i) \widehat{F} \tilde{y}(i)\right. \\
& \left.+\widetilde{u}^{T}(i) \widehat{H} \widetilde{u}(i)\right],
\end{aligned}
$$

where $\widehat{F}=\operatorname{diag}(F, F, \ldots, F) \in R^{N m \times N m}$ and $\widehat{H}=\operatorname{diag}\left(N_{1} H\right.$, $\left.N_{1} H, \ldots, N_{1} H\right) \in R^{p r \times p r}$.

Now the problem becomes an optimal regulator problem of system (18) under the performance index (19).

Substituting $\widetilde{y}(i)=\widehat{C} \widetilde{x}(i)+\widehat{D} \widetilde{u}(i)$ into (19), we get

$$
\begin{aligned}
J & =\sum_{i=0}^{\infty}\left[[\widehat{C} \tilde{x}(i)+\widehat{D} \widetilde{u}(i)]^{T} \widehat{F}[\widehat{C} \tilde{x}(i)+\widehat{D} \widetilde{u}(i)]\right. \\
& \left.+\widetilde{u}^{T}(i) \widehat{H} \widetilde{u}(i)\right]=\sum_{i=1}^{\infty}\left[\begin{array}{l}
\widetilde{x}(i) \\
\widetilde{u}(i)
\end{array}\right]^{T} \\
& \cdot\left[\begin{array}{ll}
\widehat{C}^{T} \widehat{F} \widehat{C} & \widehat{C}^{T} \widehat{F} \widehat{D} \\
\widehat{D}^{T} \widehat{F} \widehat{C} & \widehat{H}+\widehat{D}^{T} \widehat{F} \widehat{D}
\end{array}\right]\left[\begin{array}{l}
\tilde{x}(i) \\
\widetilde{u}(i)
\end{array}\right] .
\end{aligned}
$$

Because of $\widehat{F}>0$ and $\widehat{H}>0$, we have $\widehat{H}+\widehat{D}^{T} \widehat{F} \widehat{D}>0$ and

$$
\begin{aligned}
& {\left[\begin{array}{ll}
\widehat{C}^{T} \widehat{F} \widehat{C} & \widehat{C}^{T} \widehat{F} \widehat{D} \\
\widehat{D}^{T} \widehat{F} \widehat{C} & \widehat{H}+\widehat{D}^{T} \widehat{F} \widehat{D}
\end{array}\right]=\left[\begin{array}{ll}
0 & 0 \\
0 & \widehat{H}
\end{array}\right]+\left[\begin{array}{l}
\widehat{C}^{T} \\
\widehat{D}^{T}
\end{array}\right] \widehat{F}\left[\begin{array}{ll}
\widehat{C} & \widehat{D}
\end{array}\right]} \\
& \geq 0 .
\end{aligned}
$$

Further, by means of contract transformation in the matrix, we can eliminate the cross term of $\widetilde{x}(i)$ and $\widetilde{u}(i)$ in (20); that is,

$$
\begin{aligned}
& {\left[\begin{array}{ll}
\widehat{C}^{T} \widehat{F} \widehat{C} & \widehat{C}^{T} \widehat{F} \widehat{D} \\
\widehat{D}^{T} \widehat{F} \widehat{C} & \widehat{H}+\widehat{D}^{T} \widehat{F} \widehat{D}
\end{array}\right]=\left[\begin{array}{ccc}
I & 0 \\
{\left[\widehat{H}+\widehat{D}^{T} \widehat{F} \widehat{D}\right]^{-1} \widehat{D}^{T} \widehat{F} \widehat{C}} & I
\end{array}\right]^{T}} \\
& \times\left[\begin{array}{cc}
\widehat{C}^{T} \widehat{F} \widehat{C}-\widehat{C}^{T} \widehat{F} \widehat{D}\left[\widehat{H}+\widehat{D}^{T} \widehat{F} \widehat{D}\right]^{-1} \widehat{D}^{T} \widehat{F} \widehat{C} & 0 \\
0 & \widehat{H}+\widehat{D}^{T} \widehat{F} \widehat{D}
\end{array}\right] \\
& \times\left[\begin{array}{cr}
I & 0 \\
{\left[\widehat{H}+\widehat{D}^{T} \widehat{F} \widehat{D}\right]^{-1} \widehat{D}^{T} \widehat{F} \widehat{C}} & I
\end{array}\right] .
\end{aligned}
$$


Thus (20) becomes

$$
\begin{aligned}
J= & \sum_{i=1}^{\infty}\left[\tilde{x}^{T}(i)\right. \\
& +\left\{\widehat{C}^{T} \widehat{F} \widehat{C}-\widehat{C}^{T} \widehat{F} \widehat{D}\left[\widehat{H}+\widehat{D}^{T} \widehat{F} \widehat{D}\right]^{-1} \widehat{D}^{T} \widehat{F} \widehat{C}\right\} \widetilde{x}(i) \\
& +\left[\widetilde{u}(i)+\left[\widehat{H}+\widehat{D}^{T} \widehat{F} \widehat{D}\right]^{-1} \widehat{D}^{T} \widehat{F} \widehat{C} \widetilde{x}(i)\right]^{T} \times(\widehat{H} \\
& \left.\left.+\widehat{D}^{T} \widehat{F} \widehat{D}\right)\left[\widetilde{u}(i)+\left[\widehat{H}+\widehat{D}^{T} \widehat{F} \widehat{D}\right]^{-1} \widehat{D}^{T} \widehat{F} \widehat{C} \widetilde{x}(i)\right]\right] .
\end{aligned}
$$

As we all know, contract transformation cannot change the positive definiteness of the matrix. So $\widehat{C}^{T} \widehat{F} \widehat{C}-\widehat{C}^{T} \widehat{F} \widehat{D}[\widehat{H}+$ $\left.\widehat{D}^{T} \widehat{F} \widehat{D}\right]^{-1} \widehat{D}^{T} \widehat{F} \widehat{C} \geq 0$ and $\widehat{H}+\widehat{D}^{T} \widehat{F} \widehat{D}>0$.

Now the problem becomes an optimal control problem of the augmented system (18) under the performance index (23).

By using the optimal regulator theory in [10], the following theorem is obtained.

Theorem 5. If the discrete-time system (1) satisfies assumptions (A1)-(A6), the optimal output regulator is

$$
\begin{aligned}
& u\left(i N+(j-1) N_{1}\right)=K_{(j)} x(i N), \\
& u\left(i N+(j-1) N_{1}+q\right)=u\left(i N+(j-1) N_{1}\right) \\
& \left(i=0,1,2, \ldots, j=1,2, \ldots, p, q=0,1, \ldots, N_{1}-1\right),
\end{aligned}
$$

where

$$
\begin{aligned}
K & =\left[\begin{array}{c}
K_{(1)} \\
K_{(2)} \\
\vdots \\
K_{(p)}
\end{array}\right], \\
K & =-\left[\widehat{H}+\widehat{D}^{T} \widehat{F} \widehat{D}+\widehat{B}^{T} P \widehat{B}\right]^{-1} \\
& \cdot \widehat{B}^{T} P\left(\widehat{A}-\widehat{B}\left[\widehat{H}+\widehat{D}^{T} \widehat{F} \widehat{D}\right]^{-1} \widehat{D}^{T} \widehat{F} \widehat{C}\right) \\
& -\left[\widehat{H}+\widehat{D}^{T} \widehat{F} \widehat{D}\right]^{-1} \widehat{D}^{T} \widehat{F} \widehat{C}
\end{aligned}
$$

and $P$ is the unique semipositive definite solution of the algebraic Riccati equation:

$$
\begin{aligned}
P=\left(\widehat{C}^{T} \widehat{F} \widehat{C}-\widehat{C}^{T} \widehat{F} \widehat{D}\left[\widehat{H}+\widehat{D}^{T} \widehat{F} \widehat{D}\right]^{-1} \widehat{D}^{T} \widehat{F} \widehat{C}\right) \\
+\left(\widehat{A}-\widehat{B}\left[\widehat{H}+\widehat{D}^{T} \widehat{F} \widehat{D}\right]^{-1} \widehat{D}^{T} \widehat{F} \widehat{C}\right)^{T} \\
\\
+P\left(\widehat{A}-\widehat{B}\left[\widehat{H}+\widehat{D}^{T} \widehat{F} \widehat{D}\right]^{-1} \widehat{D}^{T} \widehat{F} \widehat{C}\right)
\end{aligned}
$$

$$
\begin{aligned}
& -\left(\widehat{A}-\widehat{B}\left[\widehat{H}+\widehat{D}^{T} \widehat{F} \widehat{D}\right]^{-1} \widehat{D}^{T} \widehat{F} \widehat{C}\right)^{T} \\
& \cdot P \widehat{B}\left[\widehat{H}+\widehat{D}^{T} \widehat{F} \widehat{D}+\widehat{B}^{T} P \widehat{B}\right]^{-1} \widehat{B}^{T} P \\
& \times\left(\widehat{A}-\widehat{B}\left[\widehat{H}+\widehat{D}^{T} \widehat{F} \widehat{D}\right]^{-1} \widehat{D}^{T} \widehat{F} \widehat{C}\right) .
\end{aligned}
$$

\section{Proof of Theorem 5}

To prove Theorem 5, the following two lemmas are needed.

Lemma 6. If (A5) holds, $\left(\widehat{A}-\widehat{B}\left[\widehat{H}+\widehat{D}^{T} \widehat{F} \widehat{D}\right]^{-1} \widehat{D}^{T} \widehat{F} \widehat{C} \widehat{B}\right)$ is stabilizable.

Proof. First, we prove that $\left(\widehat{A}-\widehat{B}\left[\widehat{H}+\widehat{D}^{T} \widehat{F} \widehat{D}\right]^{-1} \widehat{D}^{T} \widehat{F} \widehat{C} \widehat{B}\right)$ is stabilizable if and only if $(\widehat{A} \widehat{B})$ is stabilizable.

Noticing that elementary transformation of the matrix cannot change the rank of the matrix, and applying the $\mathrm{PBH}$ test, we have

$$
\begin{aligned}
& \operatorname{rank}\left[\lambda I-\left(\widehat{A}-\widehat{B}\left[\widehat{H}+\widehat{D}^{T} \widehat{F} \widehat{D}\right]^{-1} \widehat{D}^{T} \widehat{F} \widehat{C}\right) \widehat{B}\right] \\
& =\operatorname{rank}\left([\lambda I-\widehat{A} \widehat{B}]\left[\begin{array}{cc}
I_{n} & 0 \\
{\left[\widehat{H}+\widehat{D}^{T} \widehat{F} \widehat{D}\right]^{-1} \widehat{D}^{T} \widehat{F} \widehat{C}} & I_{p r}
\end{array}\right]\right) \\
& =\operatorname{rank}[\lambda I-\widehat{A} \widehat{B}] .
\end{aligned}
$$

So $\left(\widehat{A}-\widehat{B}\left[\widehat{H}+\widehat{D}^{T} \widehat{F} \widehat{D}\right]^{-1} \widehat{D}^{T} \widehat{F} \widehat{C} \widehat{B}\right)$ is stabilizable if and only if $(\widehat{A} \widehat{B})$ is stabilizable.

Next, we prove that if (A5) holds, $(\widehat{A} \widehat{B})$ is stabilizable.

Noticing that $\widehat{A}=A^{N}, \widehat{B}=$ $\left[\begin{array}{llll}A^{N-N_{1}} B_{p} & A^{N-2 N_{1}} B_{p} & \cdots & B_{p}\end{array}\right]$ and $B_{p}=A^{N_{1}-1} B+A^{N_{1}-2} B+$ $\cdots+B$. According to Lemma 2 , if $(A B)$ is stabilizable, for any $\lambda(|\lambda| \geq 1)$ and $w$ such that $w^{T} A=\lambda w^{T}, w^{T} B \neq 0$.

Obviously, we can derive

$$
\begin{aligned}
& w^{T} A^{i}=\lambda^{i} w^{T}, \\
& w^{T} A^{i} B=\lambda^{i}\left(w^{T} B\right) \neq 0, \\
& \quad(i=1,2, \ldots) .
\end{aligned}
$$


So

$$
\begin{aligned}
& w^{T} \widehat{A}=w^{T} A^{N}=\lambda^{N} w^{T}, \\
& w^{T} B_{p}=\left(1+\lambda+\cdots+\lambda^{N_{1}-1}\right) w^{T} B, \\
& w^{T} \widehat{B}=w^{T}\left[\begin{array}{lllll}
A^{N-N_{1}} B_{p} & A^{N-2 N_{1}} B_{p} & \cdots & B_{p}
\end{array}\right] \\
& =\left[\begin{array}{lllll}
\lambda^{N-N_{1}}\left(w^{T} B_{p}\right) & \lambda^{N-2 N_{1}}\left(w^{T} B_{p}\right) & \cdots & w^{T} B_{p}
\end{array}\right] \\
& =\left(1+\lambda+\cdots+\lambda^{N_{1}-1}\right)
\end{aligned}
$$

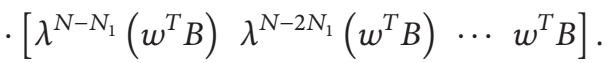

If $1+\lambda+\cdots+\lambda^{N_{1}-1} \neq 0, w^{T} \widehat{B} \neq 0$ is obtained. Using Lemma 2 again, $(\widehat{A} \widehat{B})$ is stabilizable. This completes the proof.

Lemma 7. One has the following:

$$
\left(\left\{\widehat{C}^{T} \widehat{F} \widehat{C}-\widehat{C}^{T} \widehat{F} \widehat{D}\left[\widehat{H}+\widehat{D}^{T} \widehat{F} \widehat{D}\right]^{-1} \widehat{D}^{T} \widehat{F} \widehat{C}\right\}^{1 / 2} \widehat{A}-\widehat{B}\left[\widehat{H}+\widehat{D}^{T} \widehat{F} \widehat{D}\right]^{-1} \widehat{D}^{T} \widehat{F} \widehat{C}\right)
$$

is detectable if and only if $(C A)$ is detectable.

Proof. Set $M=\widehat{C}^{T} \widehat{F} \widehat{C}-\widehat{C}^{T} \widehat{F} \widehat{D}\left[\widehat{H}+\widehat{D}^{T} \widehat{F} \widehat{D}\right]^{-1} \widehat{D}^{T} \widehat{F} \widehat{C}$ and introduce the complex unit $i=\sqrt{-1}$. Assuming

$$
V=\left[i\left\{\left[\widehat{H}+\widehat{D}^{T} \widehat{F} \widehat{D}\right]^{-1}\right\}^{1 / 2} \widehat{D}^{T} \widehat{F} \widehat{C}\right],
$$

we have $M=V^{T} V$, where $\widehat{F}=\operatorname{diag}(F, F, \ldots, F)$ and $F>0$.

So $\left(M^{1 / 2} \widehat{A}-\widehat{B}\left[\widehat{H}+\widehat{D}^{T} \widehat{F} \widehat{D}\right]^{-1} \widehat{D}^{T} \widehat{F} \widehat{C}\right)$ is detectable if and only if $\left(V \widehat{A}-\widehat{B}\left[\widehat{H}+\widehat{D}^{T} \widehat{F} \widehat{D}\right]^{-1} \widehat{D}^{T} \widehat{F} \widehat{C}\right)$ is detectable.

\section{Because}

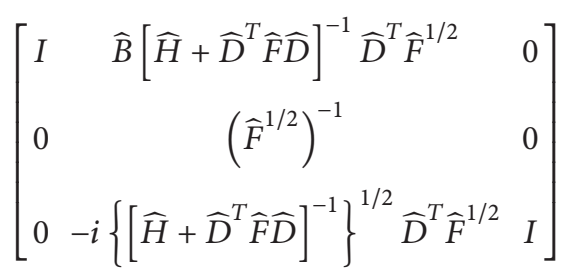

$$
\left[\begin{array}{ccc}
I & \widehat{B}\left[\widehat{H}+\widehat{D}^{T} \widehat{F} \widehat{D}\right]^{-1} \widehat{D}^{T} \widehat{F}^{1 / 2} & 0 \\
0 & \left(\widehat{F}^{1 / 2}\right)^{-1} & 0 \\
0 & -i\left\{\left[\widehat{H}+\widehat{D}^{T} \widehat{F} \widehat{D}\right]^{-1}\right\}^{1 / 2} \widehat{D}^{T} \widehat{F}^{1 / 2} & I
\end{array}\right]\left[\begin{array}{c}
\lambda I-\left(\widehat{A}-\widehat{B}\left[\widehat{H}+\widehat{D}^{T} \widehat{F} \widehat{D}\right]^{-1} \widehat{D}^{T} \widehat{F} \widehat{C}\right) \\
\widehat{F}^{1 / 2} \widehat{C} \\
i\left\{\left[\widehat{H}+\widehat{D}^{T} \widehat{F} \widehat{D}\right]^{-1}\right\}^{1 / 2} \widehat{D}^{T} \widehat{F} \widehat{C}
\end{array}\right]=\left[\begin{array}{c}
\frac{\lambda I-\widehat{A}}{\widehat{C}} \\
0
\end{array}\right],
$$

we have

$$
\begin{aligned}
& \operatorname{rank}\left[\frac{\lambda I-\left(\widehat{A}-\widehat{B}\left[\widehat{H}+\widehat{D}^{T} \widehat{F} \widehat{D}^{-1} \widehat{D}^{T} \widehat{F} \widehat{C}\right)\right.}{V}\right] \\
& \quad\left[\begin{array}{c}
\lambda I-\left(\widehat{A}-\widehat{B}\left[\widehat{H}+\widehat{D}^{T} \widehat{F} \widehat{D}\right]^{-1} \widehat{D}^{T} \widehat{F} \widehat{C}\right) \\
0
\end{array}\right] \\
& \quad \operatorname{rank}\left[\begin{array}{c}
\lambda I-\widehat{A} \\
\widehat{F} \widehat{C}^{1 / 2} \widehat{C} \\
i\left\{\left[\widehat{H}+\widehat{D}^{T} \widehat{F} \widehat{D}\right]^{-1}\right\}^{1 / 2} \widehat{D}^{T} \widehat{F} \widehat{C}
\end{array}\right]
\end{aligned}
$$


Therefore,

$$
\left(\left\{\widehat{C}^{T} \widehat{F} \widehat{C}-\widehat{C}^{T} \widehat{F} \widehat{D}\left[\widehat{H}+\widehat{D}^{T} \widehat{F} \widehat{D}\right]^{-1} \widehat{D}^{T} \widehat{F} \widehat{C}\right\}^{1 / 2} \widehat{A}-\widehat{B}\left[\widehat{H}+\widehat{D}^{T} \widehat{F} \widehat{D}\right]^{-1} \widehat{D}^{T} \widehat{F} \widehat{C}\right)
$$

is detectable if and only if $(\widehat{C} \widehat{A})$ is detectable.

And notice

$$
\begin{aligned}
& \widehat{A}=A^{N}, \\
& \widehat{C}=\left[\begin{array}{c}
C \\
\vdots \\
C A^{N_{1}-1} \\
\hline C A^{N_{1}} \\
\vdots \\
C A^{2 N_{1}-1} \\
\vdots \\
\hline C A^{(p-1) N_{1}} \\
\vdots \\
C A^{N-1}
\end{array}\right]=\left[\begin{array}{c}
C \\
C A \\
\vdots \\
C A^{N-1}
\end{array}\right] .
\end{aligned}
$$

Applying the result of [22], $(\widehat{C} \widehat{A})$ is detectable if and only if ( $C A$ ) is detectable. This completes the proof.

Proof of Theorem 5. In Section 3.2, the output regulation problem of system (1) with dual-rate setting (satisfying (A1)(A4)) has been transformed into an optimal control problem of the augmented system (18) with the performance index (23).

According to Lemmas 6 and 7, if (A5) and (A6) hold, the optimal control input of system (18) minimizing the performance index (23) is

$$
\tilde{u}(i)=K \tilde{x}(i),
$$

where $K$ is determined by (27) and $P$ is the unique semipositive definite solution of the algebraic Riccati equation (28).

According to (26) and noticing

$$
\tilde{u}(i)=\left[\begin{array}{c}
u(i N) \\
u\left(i N+N_{1}\right) \\
\vdots \\
u\left(i N+(p-1) N_{1}\right)
\end{array}\right],
$$

(39) can be written as

$$
\left[\begin{array}{c}
u(i N) \\
u\left(i N+N_{1}\right) \\
\vdots \\
u\left(i N+(p-1) N_{1}\right)
\end{array}\right]=\left[\begin{array}{c}
K_{(1)} \\
K_{(2)} \\
\vdots \\
K_{(p)}
\end{array}\right] x(i N) .
$$

The above equation can be simplified to (24).

And due to (A4),

$$
\begin{aligned}
& u\left(i N+(j-1) N_{1}+q\right)=u\left(i N+(j-1) N_{1}\right) \\
& \left(i=0,1,2, \ldots, j=1,2, \ldots, p, q=0,1, \ldots, N_{1}-1\right) .
\end{aligned}
$$

\section{Numerical Example}

In this section, a numerical example is included to illustrate the effectiveness of the proposed method.

Consider a linear discrete-time system:

$$
\begin{aligned}
x(k+1) & =\left[\begin{array}{cc}
-0.62 & 0.52 \\
1.1 & 0.4
\end{array}\right] x(k)+\left[\begin{array}{c}
-0.6 \\
0.18
\end{array}\right] u(k), \\
y(k) & =[-0.3-1.5] x(k) .
\end{aligned}
$$

In comparison with system (1), the coefficient matrices are as follows:

$$
\begin{aligned}
& A=\left[\begin{array}{cc}
-0.62 & 0.52 \\
1.1 & 0.4
\end{array}\right], \\
& B=\left[\begin{array}{c}
-0.6 \\
0.18
\end{array}\right], \\
& C=\left[\begin{array}{ll}
-0.3 & -1.5
\end{array}\right] .
\end{aligned}
$$

Suppose $N=4$ and $N_{1}=2$; that is, the state vector $x(k)$ and output vector $y(k)$ can only be measured at $k=4 i$ and the input vector $u(k)$ can be input only at $k=2 i(i=0,1,2, \ldots)$. Let the initial conditions be $x(0)=\left[\begin{array}{l}0.2 \\ 1.5\end{array}\right], u(0)=0$, and $u(2)=$ 0.5 . In addition, take the weight matrices $F=1$ and $H=100$.

Through verifying, all of the conditions of Theorem 5 are satisfied. According to Theorem 5, we obtain the feedback matrix:

$$
K=\left[\begin{array}{ll}
-5.9905 & 2.1971 \\
-5.7141 & 2.0930
\end{array}\right]
$$

Next we perform MATLAB simulation results. The output response of the discrete-time system with dual-rate setting is shown in Figure 1 and the corresponding control input is shown in Figure 2. In the upper right of these two figures, there are magnified simulated images around $t=60$. Note that the output response of the system can reach steady state rapidly from Figure 1, and the designed optimal output regulator is effective. The input curve of the closed-loop system is shown in Figure 2.

We notice that the output response in Figure 1 is not quite smooth. The response shows a small oscillation, which is the multirate feature of the system. In addition, the input curve shows a stair-step feature in Figure 2. This is because $\mathrm{ZOH}$ is used in the input. 


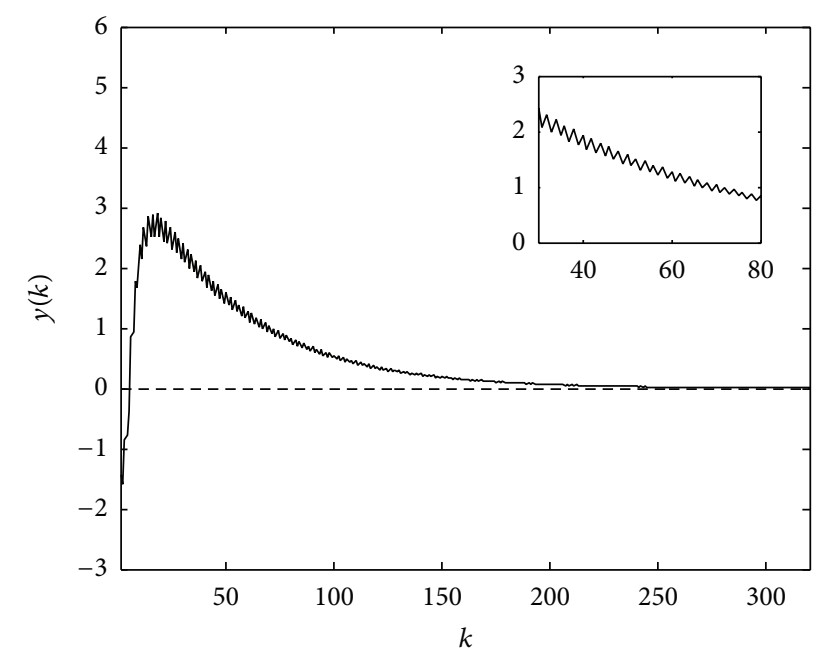

FIGURE 1: The output response of the dual-rate discrete-time system.

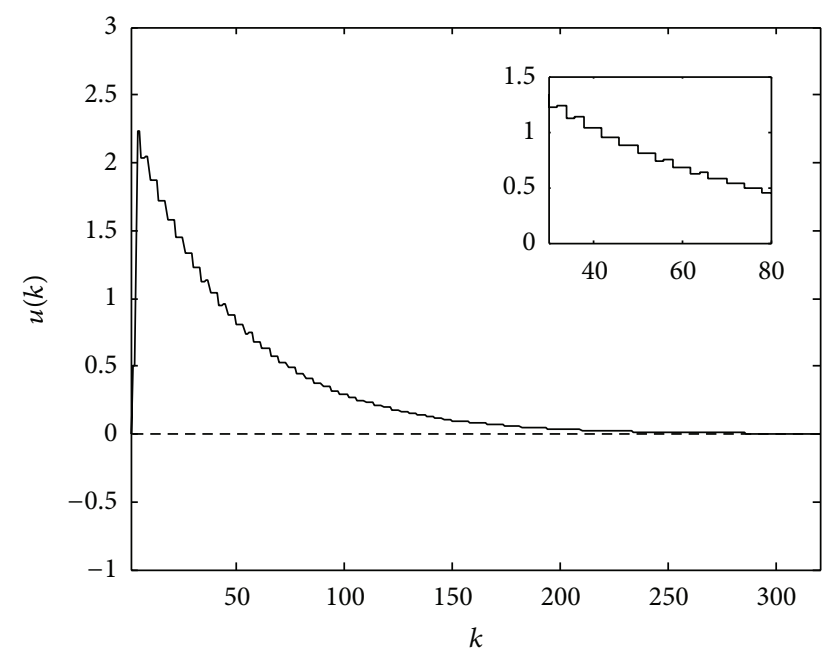

FIgURE 2: The control input of the dual-rate discrete-time system.

\section{Conclusion}

In this paper, we studied the optimal output regulator for a class of linear discrete-time systems with dual-rate setting. By using the discrete lifting technique, the dual-rate discretetime system is converted to a single-rate augmented system in form, and the lifted state-space model is constructed. Then we can use the method for single-rate systems to study the optimal regulator problem of the lifted system. By using optimal regulator theory, an optimal output regulator for the dual-rate discrete-time system is finally derived. This approach is also a guideline for future study of the optimal preview control problem for dual-rate systems. Furthermore, when assumption (A3) does not hold, for example, in the cases where $N / N 1$ is not an integer or $N 1 / N>1$, this approach can also be applied. But the lifting process is different and the corresponding lifted system need to be reconstructed. Finally, the numerical simulation showed the effectiveness and validity of the conclusions in this paper.

\section{Competing Interests}

The authors declare that there are no competing interests regarding the publication of this paper.

\section{Acknowledgments}

This work is supported by the National Natural Science Foundation of China (no. 61174209).

\section{References}

[1] J. Xiao, Multirate Sampling Digital Control System, Science Press, Beijing, China, 2003.

[2] F. Ding, T.-W. Chen, and D.-Y. Xiao, "State-space modeling and identification of general dual-rate stochastic systems," Acta Automatica Sinica, vol. 30, no. 5, pp. 652-663, 2004.

[3] D. Li, S. L. Shah, T. Chen, and K. Z. Qi, "Application of dualrate modeling to CCR octane quality inferential control," IEEE Transactions on Control Systems Technology, vol. 11, no. 1, pp. 43-51, 2003.

[4] J. Salt and M. Tomizuka, "Hard disk drive control by model based dual-rate controller. Computation saving by interlacing," Mechatronics, vol. 24, no. 6, pp. 691-700, 2014.

[5] J. Sheng, T. Chen, and S. L. Shah, "Optimal filtering for multirate systems," IEEE Transactions on Circuits and Systems II: Express Briefs, vol. 52, no. 4, pp. 228-232, 2005.

[6] Y. Zou, T. Chen, and S. Li, "Network-based predictive control of multirate systems," IET Control Theory and Applications, vol. 4, no. 7, pp. 1145-1156, 2010.

[7] W. Chen and L. Qiu, "Stabilization of networked control systems with multirate sampling," Automatica, vol. 49, no. 6, pp. 15281537, 2013.

[8] B. Zhang, K. Zhou, and D. Wang, "Multirate repetitive control for PWM DC/AC converters," IEEE Transactions on Industrial Electronics, vol. 61, no. 6, pp. 2883-2890, 2014.

[9] S. López-López, A. Sideris, and J. Yu, "Two-stage $H_{\infty}$ optimization approach to multirate controller design," International Journal of Control, Automation and Systems, vol. 10, no. 4, pp. 675-683, 2012.

[10] C. Li and G. Ma, Optimal Control, Science Press, Beijing, China, 2011.

[11] K. G. Arvanitis, P. N. Paraskevopoulos, and A. A. Vernardos, "Multirate adaptive temperature control of greenhouses," Computers and Electronics in Agriculture, vol. 26, no. 3, pp. 303-320, 2000.

[12] Y. Zhang, Z. Sun, and J. Xiao, "Design of multi-rate optimal regulator for an inverted pendulum," Journal of Southwest Jiao Tong University, vol. 37, no. 3, pp. 281-285, 2002.

[13] Q.-S. Shi and F.-C. Liao, "Design of an optimal preview controller for linear discrete-time multirate systems with statedelay," Journal of University of Science and Technology Beijing, vol. 33, no. 3, pp. 363-375, 2011.

[14] F. Liao, M. Tomizuka, M. Cao, and D. Wang, "Optimal preview control for discrete-time descriptor causal systems in a multirate setting," International Journal of Control, vol. 86, no. 5, pp. 844-854, 2013.

[15] M. Cao and F. Liao, "Design of an optimal preview controller for linear discrete-time descriptor noncausal multirate systems," The Scientific World Journal, vol. 2014, Article ID 965915, 11 pages, 2014. 
[16] K. G. Arvanitis and G. Kalogeropoulos, "Stability robustness of LQ optimal regulators based on multirate sampling of plant output," Journal of Optimization Theory and Applications, vol. 97, no. 2, pp. 299-337, 1998.

[17] M.-J. Cao and F.-C. Liao, "Design of optimal output regulators for multirate linear discrete-time descriptor causal system with time delay," Journal of University of Science and Technology Beijing, vol. 36, no. 4, pp. 551-561, 2014.

[18] D. Z. Zheng, Linear Systems Theory, Tsinghua University Press, Beijing, China, 2002.

[19] K. Zhou, J. C. Doyle, and K. Glover, Robust and Optimal Control, Prentice Hall, Englewood Cliffs, NJ, USA, 1996.

[20] T. Chen and B. A. Francis, Optimal Sampled-Data Control Systems, Springer, New York, NY, USA, 1995.

[21] A. K. Tangirala, D. Li, R. Patwardhan, S. L. Shah, and T. Chen, "Issues in multirate process control," in Proceedings of the American Control Conference (ACC '99), pp. 2771-2775, San Diego, Calif, USA, June 1999.

[22] F. Liao, K. Takaba, T. Katayama, and J. Katsuura, "Design of an optimal preview servomechanism for discrete-time systems in a multirate setting," Dynamics of Continuous, Discrete and Impulsive Systems, Series B: Applications and Algorithms, vol. 10, no. 5, pp. 727-744, 2003. 


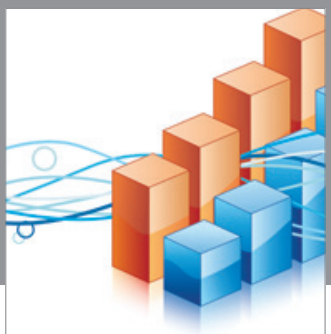

Advances in

Operations Research

vatem alat4

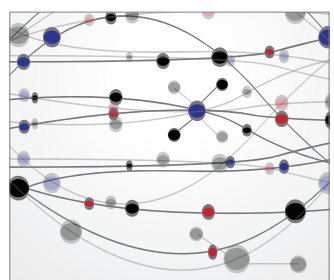

\section{The Scientific} World Journal
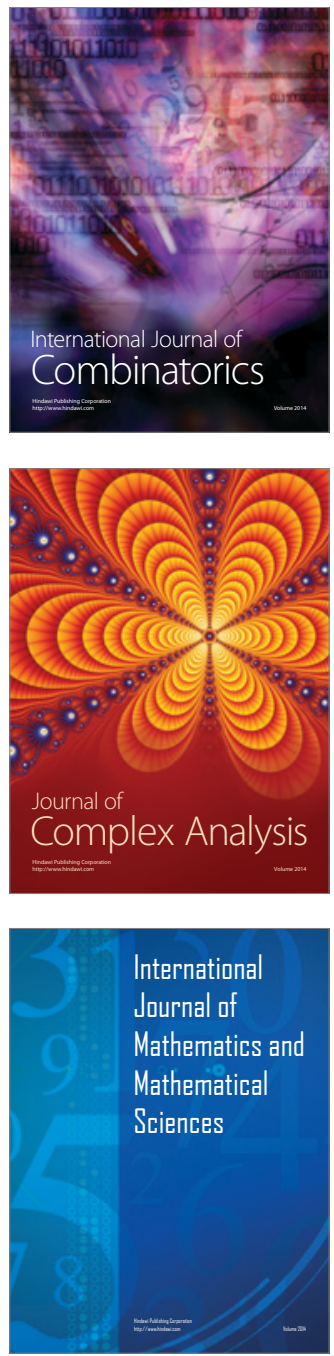
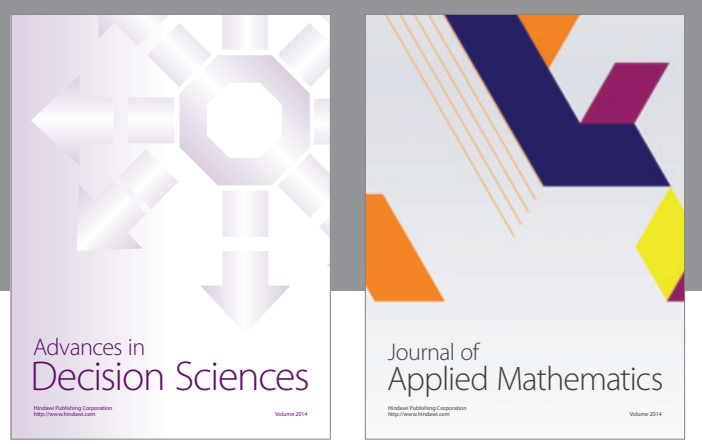

Algebra

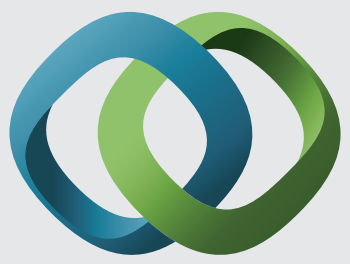

\section{Hindawi}

Submit your manuscripts at

http://www.hindawi.com
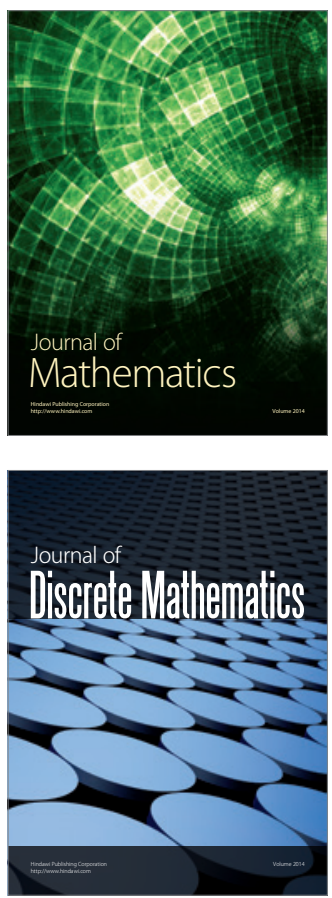

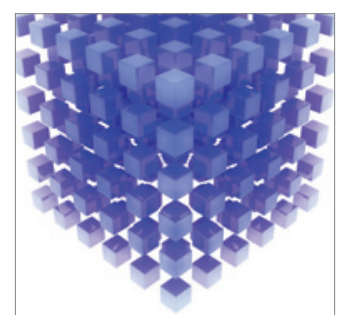

Mathematical Problems in Engineering
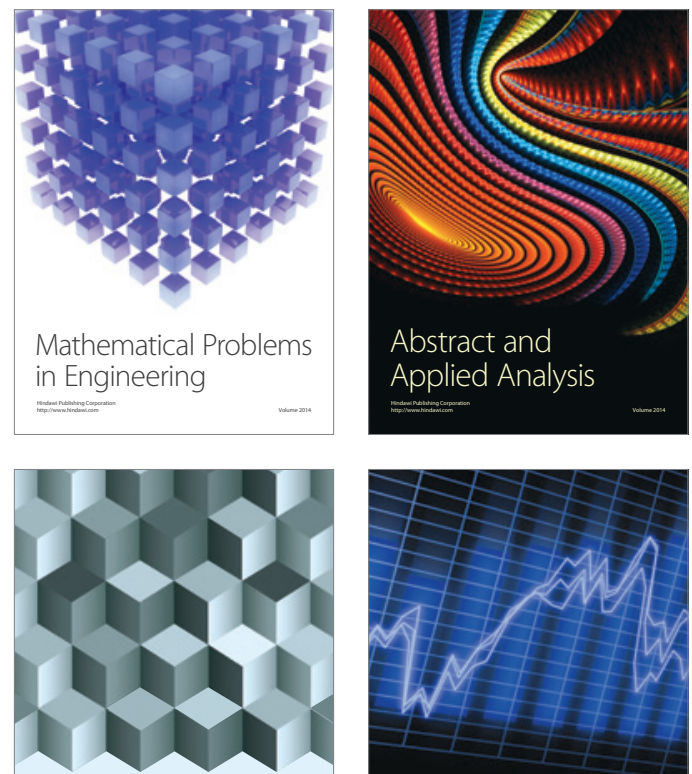

Journal of

Function Spaces

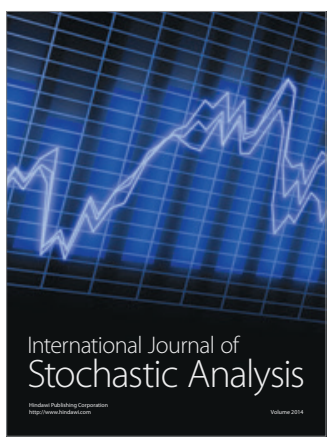

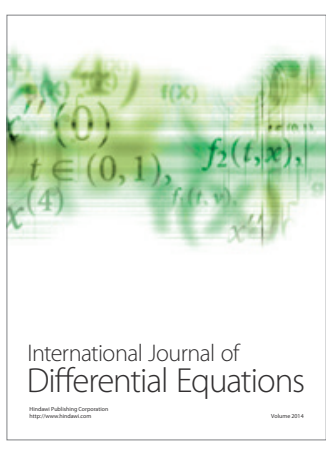
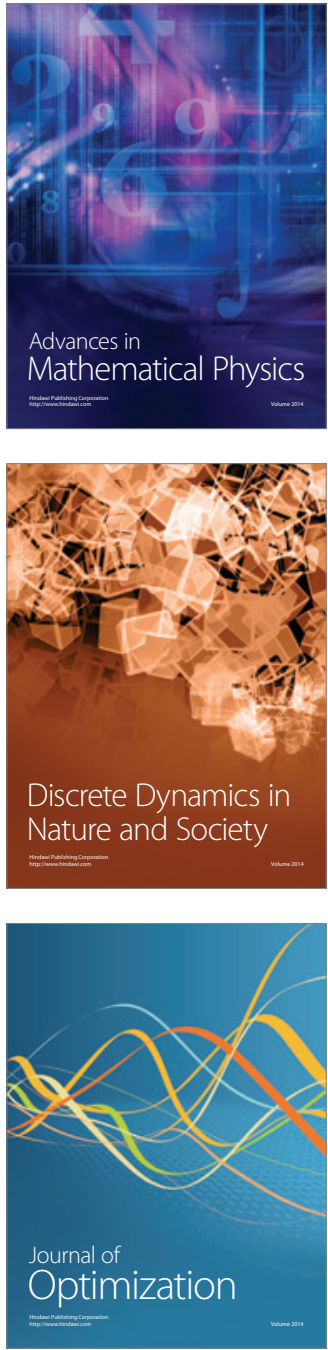